Rev. Int. Contam. Ambie. 37, 145-153, 2021

https://doi.org/10.20937/RICA.53725

\title{
RESIDUOS DE PLAGUICIDAS EN CULTIVOS DEL MUNICIPIO ZONA BANANERA, DEPARTAMENTO DEL MAGDALENA, COLOMBIA
}

Pesticide wastes in crops from the Zona Bananera municipality, Department of Magdalena, Colombia

\section{Yiniva CAMARGO CAICEDO ${ }^{1 *}$, Fredy TOVAR-BERNAL ${ }^{1}$ y Eliana ÁLVAREZ-PINEDA ${ }^{2}$}

${ }^{1}$ Grupo de Investigación en Modelación de Sistemas Ambientales, Universidad del Magdalena, Carrera 32 núm. 22-08, C.P. 470004, Santa Marta, Colombia.

${ }^{2}$ Corporación Autónoma Regional del Magdalena, Avenida del Libertador 32-201, Santa Marta, Colombia.

*Autora para correspondencia: ycamargo@unimagdalena.edu.co

(Recibido: septiembre de 2019; aceptado: julio de 2020)

Palabras clave: agroquímicos, clorpirifós, cultivos de banano, residuos peligrosos.

\section{RESUMEN}

El proceso productivo del banano se encuentra relacionado con la generación de residuos o desechos peligrosos que pueden ocasionar potencial contaminación de los recursos hídricos y del suelo, así como afectar la salud de los trabajadores, por lo que el objetivo de este trabajo consistió en identificar los principales residuos de plaguicidas asociados a las actividades de producción de banano de exportación en el municipio Zona Bananera, Departamento del Magdalena, Colombia. La metodología consistió principalmente en visitas efectuadas a 46 fincas bananeras, dos centros de acopio de residuos y un gestor de residuos peligrosos para la recolección de información primaria, sistematización y análisis de datos. De esta forma, se categorizaron los residuos peligrosos generados en las cuatro etapas del proceso productivo: siembra, manejo del cultivo, cosecha y postcosecha, identificando que en el manejo del cultivo las actividades de control de malezas, amarre, embolse y control fitosanitario, constituyen las de mayor generación de diversas tipologías de residuos, dentro de las que se pueden enumerar los recipientes vacíos de agroquímicos, las bolsas con aditivos y otros desechos peligrosos resultado del uso de elementos de protección personal por parte de los trabajadores y de la preparación y utilización de biocidas, entre otros. Se concluye que la identificación de estos residuos peligrosos contribuye a la elaboración e implementación del Plan de Gestión de Residuos Peligrosos en Zona Bananera, conducente al adecuado manejo de estos residuos por parte de los generadores y al fortalecimiento del ejercicio de control ambiental por los entes reguladores.

Key words: agrochemicals, banana crops, chlorpyrifos, hazardous wastes.

\begin{abstract}
Banana production is related to the generation of hazardous wastes that can cause potential contamination to the water, the soil and the workers' health. The objective of the present work was to identify the main pesticide wastes associated to export banana production activities from the Zona Bananera municipality, Department of Magdalena,
\end{abstract}


Colombia. The methodology consisted of systematic observation, primary data collection and data analysis of 46 banana farms, two waste collection centers, and a hazardous waste manager. In this way, the hazardous wastes generated in the four stages of banana crop was categorized in sowing, crop management, harvesting and postharvest. In the banana crop management, weed control, mooring, tagging and phytosanitary management were the activities with greater generation of hazardous wastes, among which empty containers of agrochemicals, bags with additive chlorpyrifos/biphentrine, and other hazardous waste such as personal protective elements and preparation and use of biocides. It is concluded that the identification of hazardous wastes contributes to the development and implementation of the Hazardous Waste Management Plan in the Zona Bananera municipality, Department of Magdalena, Colombia.

\section{INTRODUCCIÓN}

La producción agrícola debe enfrentar la problemática relevante asociada con el uso de plaguicidas para el control de plagas y enfermedades de los cultivos, que se evidencia a partir de sus potenciales riesgos para la salud humana y sus efectos en el ambiente (GómezArroyo et al. 2013, Guzmán-Cabrera et al. 2017).

En actividades agrícolas, un aspecto importante relacionado con el uso de plaguicidas es la generación de residuos peligrosos, definidos como aquellos que por sus características corrosivas, reactivas, explosivas, tóxicas, inflamables, infecciosas o radiactivas pueden causar riesgos, daños o efectos no deseados, directos o indirectos, a la salud humana y/o al ambiente. La norma colombiana incluye en esta categoría de residuos a los empaques, envases o embalajes que los hayan contenido, así como subproductos de plaguicidas, productos de lavado u objetos que hayan estado en contacto con éstos, i.e. ropa de trabajo, equipos de aplicación, equipos de proceso, entre otros (MADS 2015).

En el caso particular de la producción agrícola de banano, el Informe de Evaluaciones Agropecuarias del Ministerio de Agricultura y Desarrollo Rural (MADR), a través de su herramienta AgroNet, registra las estadísticas de área, producción, rendimiento y participación municipal en el Departamento del Magdalena para el cultivo de banano durante el periodo 2007-2014. De este informe es posible identificar al municipio Zona Bananera con la mayor cantidad de hectáreas cultivadas (un promedio de 8150 ha) para el periodo evaluado, así como un comportamiento relativamente constante (MADR 2016).

Zona Bananera está localizado al norte del Departamento del Magdalena, Colombia, entre las coordenadas $10^{\circ} 39^{\prime}-10^{\circ} 55^{\prime} \mathrm{N}$ y $74^{\circ} 06^{\prime}-4^{\circ} 17^{\prime} \mathrm{W}$, a una altura de $30 \mathrm{msnm}$, con $446 \mathrm{~km}^{2}$ de extensión que corresponden al $1.9 \%$ de la superficie total del departamento (Fig. 1). Es el principal municipio productor de banano con destino a la exportación en el Departamento del Magdalena. Cuenta con 47971 ha, de las cuales $76.3 \%$ tienen vocación agrícola y $22.4 \%$ pecuaria. La actividad productiva se centra en el cultivo de banano (43\%) y palma de aceite (51\%), de los que se generan $75 \%$ de los empleos a nivel local y, por ende, una alta dependencia económica (DNP 2016).

El incremento de la actividad productiva en el sector bananero del municipio ha generado el manejo inadecuado de residuos peligrosos, siendo cada

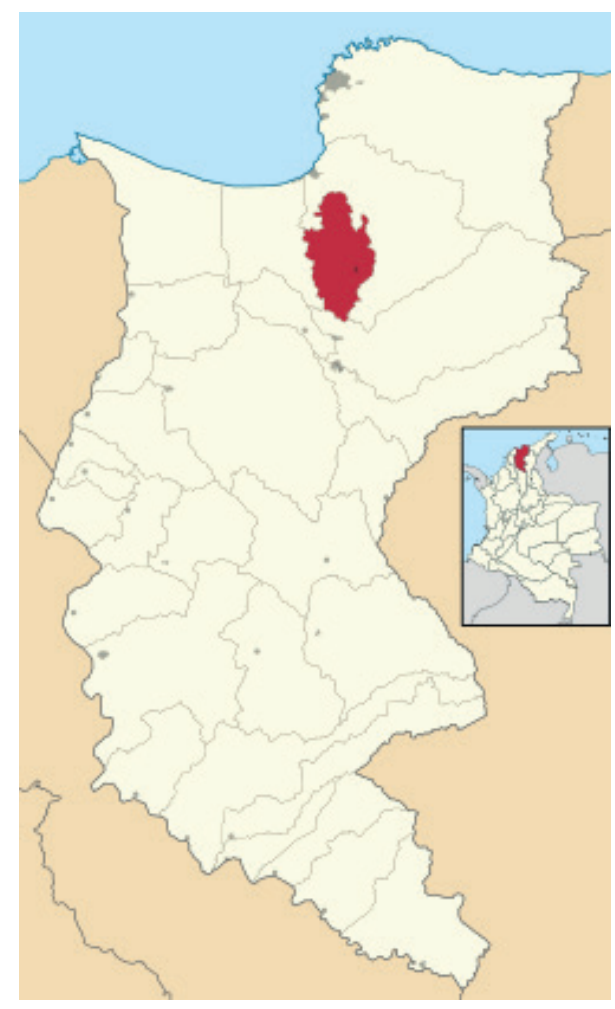

Fig. 1. Localización del municipio Zona Bananera, Departamento del Magdalena, Colombia (Alcaldía Municipal Zona Bananera 2016). 
vez más frecuente la presencia de envases vacíos de plaguicidas, bolsas con y sin aditivos, así como elementos de amarre del banano en las corrientes de agua natural de los ríos pertenecientes a la vertiente hidrográfica II del Departamento del Magdalena. Esta vertiente corresponde a los ríos que nacen y recorren la ladera sudoccidental de la Sierra Nevada de Santa Marta y desembocan en la Ciénaga Grande, y está conformada por las cuencas de los ríos Tucurinca, Sevilla y Riofrío, de caudal permanente y uso mayoritario para riego de cultivos y actividades ganaderas en el área de estudio (Alcaldía Municipal Zona Bananera 2016).

La producción de banano en Zona Bananera se realiza en cuatro etapas: siembra, manejo del cultivo, cosecha y postcosecha. Durante la etapa de cultivo es necesaria la actividad de embolse con fundas perforadas de polietileno de baja densidad (LDPE) con adición del insecticida organofosforado Dursban al 1 $\%$, con nombre genérico clorpirifós, para proteger el fruto de agentes externos como el ataque de insectos (áfidos, polillas y escarabajos), permitiendo así una mejora en la apariencia del racimo y en el llenado de la fruta. Otra alternativa para el control de insectos es el embolse utilizando fundas sin aditivos con bandas impregnadas de clorpirifós al $1 \%$ denominadas corbata o corbatín (CORPAMAG 2017).

Residuos como los generados en el proceso de embolse en la etapa de cultivo del banano son depositados en cuerpos de agua y/o suelo, al igual que otros residuos peligrosos. Lo anterior es motivo de preocupación debido al uso principal del agua de estas corrientes y a la vocación productiva del suelo en el área de estudio, ya que plantea un riesgo potencial de contaminación ambiental y afectación a las personas que pueden tener contacto directo con estos residuos, como han documentado Gómez-Arroyo et al. (2013).

En ese sentido, el objetivo de este trabajo consistió en identificar los principales residuos de plaguicidas asociados a las actividades de producción de banano de exportación en el municipio Zona Bananera, Departamento del Magdalena, Colombia.

\section{MATERIALES Y MÉTODOS}

En este trabajo se propuso un marco metodológico de referencia para identificar los principales residuos de plaguicidas asociados a las actividades de producción de banano de exportación en el municipio Zona Bananera, Departamento del Magdalena, Colombia. Éste consistió en la realización de visitas técnicas a las fincas bananeras, centros de acopio de residuos y gestores de residuos peligrosos para la recolección de información primaria mediante observación y aplicación de instrumentos de recolección de datos. Estas actividades se complementaron con la consulta de información secundaria en bases de datos bibliográficas, documentos técnicos de la autoridad ambiental local (Corporación Autónoma Regional del Magdalena [CORPAMAG]) y guías ambientales del Ministerio de Ambiente y Desarrollo Sostenible (MADS).

\section{Fuentes de información primaria y secundaria}

La recolección de información primaria de la gestión integral de residuos de plaguicidas asociados a la producción bananera en el municipio Zona Bananera, se efectuó a partir de la observación y aplicación de instrumentos de recolección de datos durante visitas técnicas a 46 fincas bananeras, dos centros de acopio de residuos y un gestor de residuos peligrosos.

Esta información se sistematizó para proceder a su revisión y respectivo análisis, obteniendo registros relacionados con el tamaño del generador de residuos (grande, mediano o pequeño), procesos y/o actividades de la producción del banano que generan residuos de plaguicidas, principales sustancias peligrosas utilizadas, corrientes de residuos asociadas a éstas, tipología de bolsa empleada en el proceso productivo (con aditivo clorpirifós o bifentrina, sin aditivo o una combinación de éstas), almacenamiento de envases vacíos de agroquímicos, entre otros.

Las características de la producción de banano de exportación en el Departamento del Magdalena se obtuvieron a partir de la información bibliográfica reportada por la Asociación de Bananeros de Colombia (AUGURA 2017). Además, se consultaron los documentos de evaluaciones agropecuarias de la base de datos AgroNet del Ministerio de Agricultura y Desarrollo Rural (MADR), la cual registra las estadísticas de área, producción, rendimiento y participación municipal del Departamento del Magdalena para el cultivo de banano.

Análisis de la información de residuos de plaguicidas asociados a las actividades de producción de banano en el municipio Zona Bananera

Se realizó el análisis de la información recolectada de fuentes primarias y secundarias conducentes a la descripción de las etapas del proceso productivo del banano de exportación: siembra, manejo del cultivo, cosecha y postcosecha, así como de los residuos peligrosos asociados a éstas, en el Departamento de Magdalena. La identificación de los agroquímicos de uso regular en las diferentes etapas de la producción 
del banano (nombre comercial, ingrediente activo, categoría toxicológica y grupo químico) también fue de utilidad para relacionarla con la tipología de residuo peligroso y corriente de residuo establecido en la norma colombiana vigente.

\section{RESULTADOS}

\section{Producción de banano de exportación en el De- partamento del Magdalena}

Las visitas técnicas a las fincas en el municipio Zona Bananera facilitaron la identificación de los residuos peligrosos asociados con las cuatro etapas del proceso: siembra, manejo del cultivo, cosecha y postcosecha (Fig. 2). Durante las observaciones efectuadas se registró que la siembra incluye la mezcla de abonos, el depósito de la semilla en el terreno previamente acondicionado con la infraestructura adecuada, una etapa libre de generación de residuos peligrosos. El manejo del cultivo comprende las actividades necesarias para la obtención de una buena producción, tales como: $i$ ) control de malezas; ii) desmanche; iii) deshoje, desguasque y destronque; $i v$ ) amarre; $v$ ) embolse; $v i$ ) fertilización, y vii) manejo fitosanitario. El amarre, el embolse y el control fitosanitario constituyen las actividades de mayor generación de residuos peligrosos. La cosecha es la etapa que inicia desde el corte de los racimos hasta su transporte a la empacadora, e implica procedimientos para conservar las características esenciales de la fruta hasta su consumo final, como el encintado que sirve para identificar la edad de los racimos y así programar la cosecha. La postcosecha comprende el alistamiento, el empaque-embalaje y el transporte de la fruta, siendo necesaria la aplicación de buenas prácticas de manejo e higiene con miras a la exportación de un producto de alta calidad e inocuidad. La cosecha y postcosecha generan residuos peligrosos de tres tipologías que se enumeran en el siguiente apartado.

\section{Residuos peligrosos generados en las etapas de producción de banano en el Departamento del Magdalena}

En la figura 3 se presentan los principales residuos peligrosos generados en el manejo de cultivo, cosecha y postcosecha del banano en el departamento del Magdalena. Las actividades más relevantes en la generación de residuos peligrosos durante el manejo de cultivo son: $i$ ) control de malezas, en la que se producen envases de herbicidas (Finale SL, Panzer 480 SL, Reglone SL, entre otros) y elementos de protección personal (EPP) contaminados; ii) amarre, que genera nylon y cintas contaminadas y recipientes de ácido giberélico; iii) embolse, en que se producen bolsas con aditivo clorpirifós y EPP contaminados; iv) manejo fitosanitario, que genera envases de fungicidas foliares (e.g., Mancozeb, Nufarm 800 WP, etc.) y recipientes de plaguicidas manuales (Lorsban al $2.5 \%$ DP, Burner $150 \mathrm{SL}$, Roundup 747, entre otros).

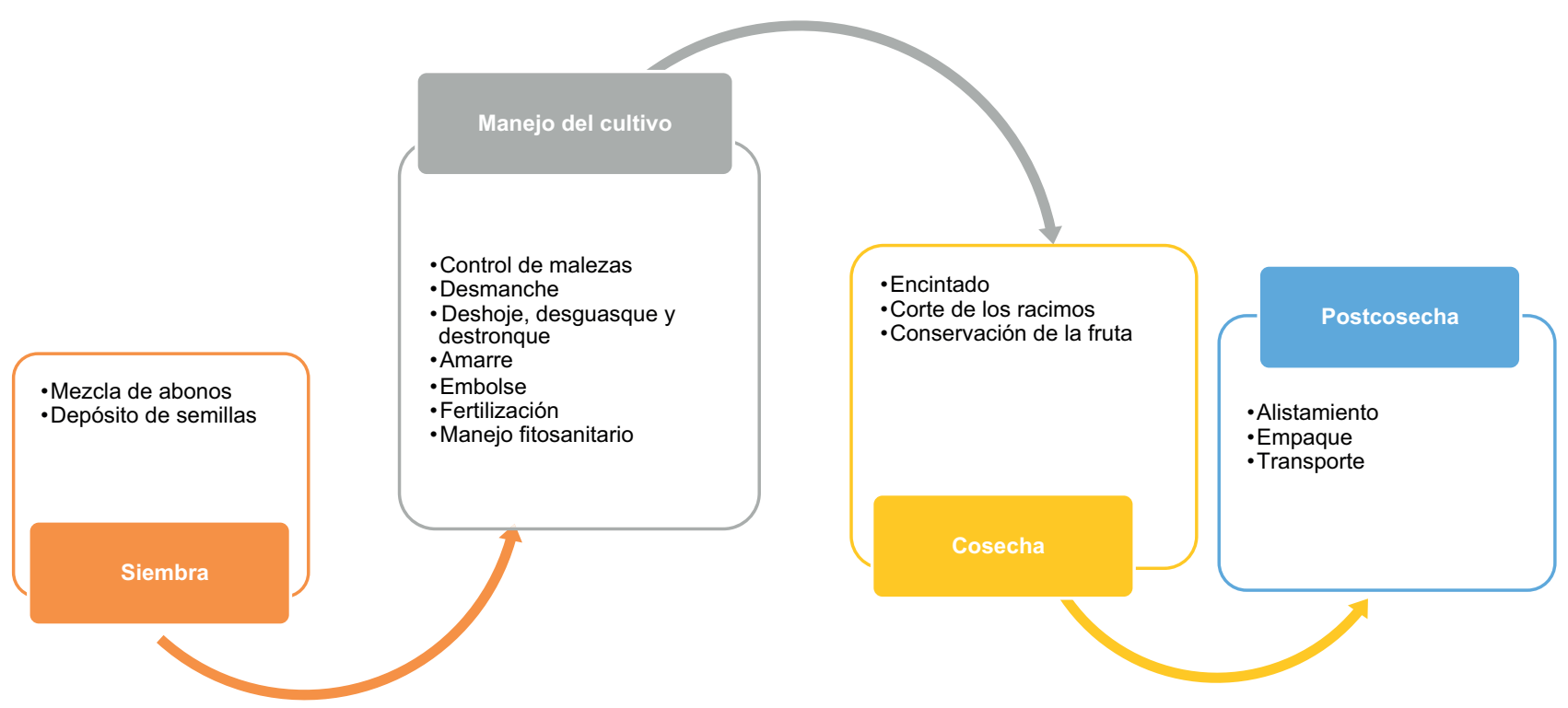

Fig. 2. Etapas del proceso productivo de banano de exportación en el Departamento del Magdalena, Colombia (Moreno et al. 2009). 


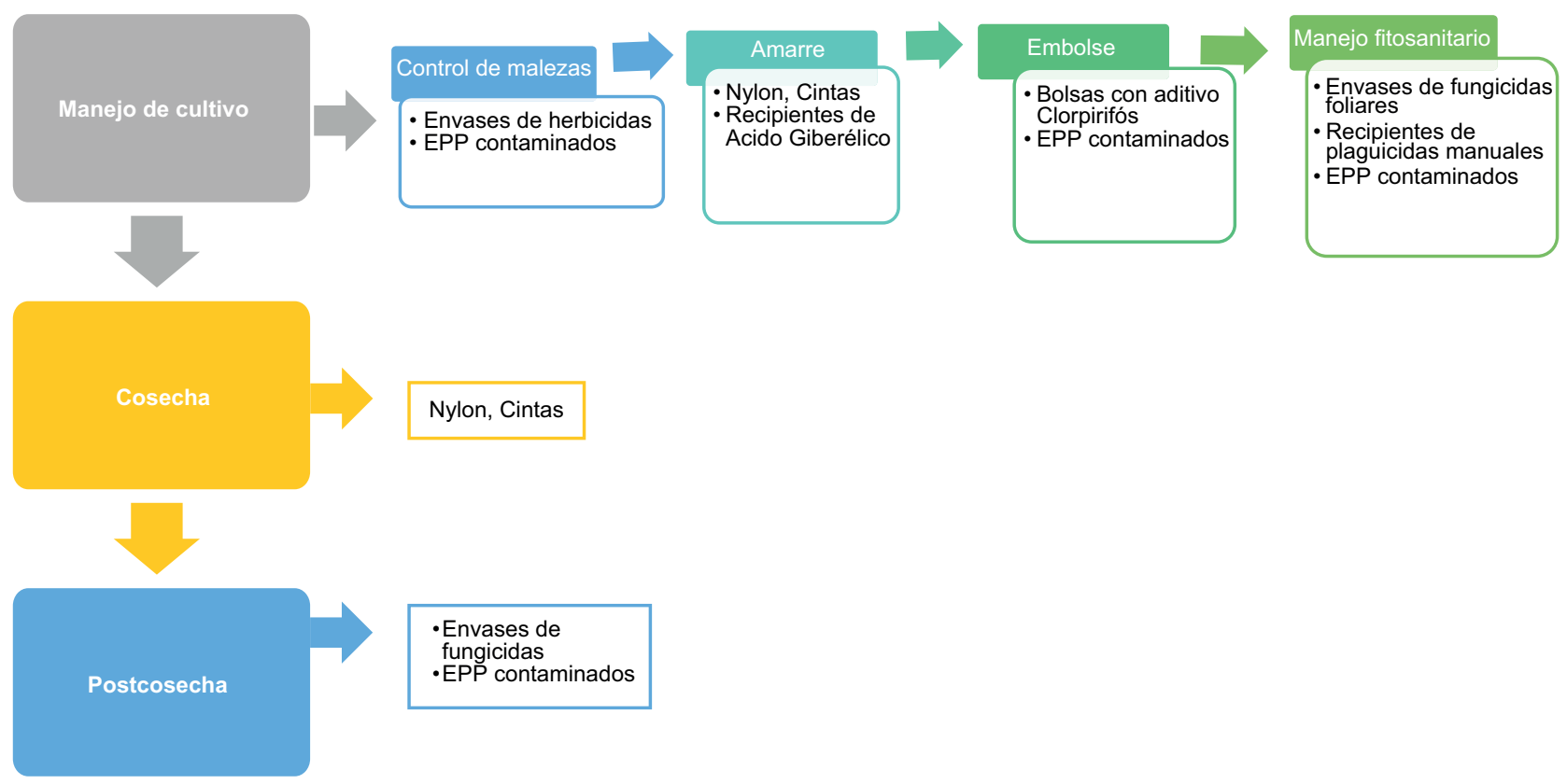

Fig. 3. Residuos peligrosos generados en las etapas de producción de banano en el departamento del Magdalena, Colombia.

La cosecha de banano produce residuos peligrosos tales como nylon y cintas contaminadas, mientras que la postcosecha genera envases de fungicidas (Magnate sulfato 75 SP, Mertect 500 SC, Helmstar 50 WG y Amistar $50 \mathrm{WG}$ ) y EPP contaminados.

La categoría de EPP contaminados se encuentra conformada por elementos utilizados en la preparación, manipulación y aplicación de herbicidas y fungicidas durante el proceso productivo, tales como los guantes de nitrilo, mascarillas, botas de caucho, delantales, overoles y gafas protectoras, entre otros.

Adicionalmente, en la etapa de fertilización se identifican residuos como recipientes de urea, sulfato de potasio, KCL, sulfato de amonio, así como yeso agrícola, que vale la pena mencionar, aunque sus características no revisten peligrosidad.

En el cuadro I se presentan los principales agroquímicos empleados en el manejo del cultivo, cosecha y postcosecha de la producción bananera del Departamento del Magdalena, indicando nombre comercial, ingrediente activo, categoría toxicológica y grupo químico. Dichos agroquímicos posteriormente generan envases vacíos clasificados mayoritariamente en la categoría toxicológica II (altamente tóxico) con 16 registros, de los cuales 12 corresponden a fungicidas aéreos, seguidos de la categoría toxicológica III (moderamente tóxico) con 15 registros, de los cuales ocho corresponden a fungicidas aéreos; final- mente, en la categoría toxicológica IV (ligeramente tóxico) se identificaron cinco registros que incluyen un fungicida aéreo.

Otros residuos peligrosos generados por procesos/ actividades o corrientes de residuo en las fincas bananeras se categorizan a partir de lo establecido en la normativa colombiana contenida en los anexos I y II del artículo 2.2.6.2.3.6 del Decreto 1076 de 2015 (Decreto Único Reglamentario del Sector Ambiente y Desarrollo Sostenible) (cuadros II y III).

Se destaca la generación de residuos peligrosos con contenido de emulsiones de agua-aceite o hidrocarburos y agua (Y9-A4060) en la actividad productiva, los cuales tienen origen variado dada la multitud de procedimientos conexos tales como mantenimiento de maquinaria y equipos industriales, mantenimiento de automotores, así como lubricación de piezas, entre otros. También se encontraron residuos de la categoría Y8-A3020 (desechos de aceites minerales o aceites usados) e Y18, correspondientes a los resultantes de la eliminación de desechos industriales (textiles y otros elementos contaminados con aceite y productos químicos, como EPP de los trabajadores).

Adicionalmente, se registraron residuos asociados con las corrientes Y4-A4030 resultantes de la producción, la preparación y la utilización de biocidas y productos fitofarmacéuticos, que cobran importancia por su carácter altamente tóxico, así 
CUADRO I. PRINCIPALES AGROQUÍMICOS UTILIZADOS EN LA PRODUCCIÓN DE BANANO EN EL MUNICIPIO ZONA BANANERA.

\begin{tabular}{|c|c|c|c|c|}
\hline $\begin{array}{l}\text { Actividad } \\
\text { biológica }\end{array}$ & Nombre comercial & Ingrediente activo & $\begin{array}{l}\text { Categoría toxico- } \\
\text { lógica }\end{array}$ & Grupo químico \\
\hline \multirow{4}{*}{$\begin{array}{l}\text { Fungicidas } \\
\text { postcosecha }\end{array}$} & Magnate sulfato $75 \mathrm{SP}$ & Imazalil & II & Imidazoles \\
\hline & Mertect $500 \mathrm{SC}$ & Tiabendazol & III & Benzimidazoles \\
\hline & Helmstar $50 \mathrm{WG}$ & Azoxystrobin & III & Estrobirulinas \\
\hline & Amistar $50 \mathrm{WG}$ & Azoxystrobina & IV & Estrobirulinas \\
\hline Fungicidas foliares & Mancozeb Nufarm 800 WP & Mancozeb & III & Ditiocarbamatos \\
\hline \multirow{3}{*}{ Herbicidas } & Reglone SL & Dibromuro de diquat & II & Bipiridilos \\
\hline & Finale SL & Glufosinato de amonio & IV & Acidos amino fosfínicos \\
\hline & Panzer 480 SL & Glifosato & IV & Organofosfonatos \\
\hline \multirow{7}{*}{ Plaguicidas } & Touchdown IQ SL & Glifosato & III & Organofosfonatos \\
\hline & Destierro & Glufosinato de amonio & II & Acidos amino fosfínicos \\
\hline & Gexapax & Ametrina & III & Triazinas \\
\hline & Roundup 747 & Glifosato & IV & Organofosfonatos \\
\hline & Burner $150 \mathrm{SL}$ & Glufosinato de amonio & II & Acidos amino fosfínicos \\
\hline & Goal 480 SC & Oxyfluorfen & III & Difenileteres \\
\hline & Lorsban $2.5 \% \mathrm{DP}$ & Clorpirifós & III & Organofosforados \\
\hline \multirow{21}{*}{$\begin{array}{l}\text { Fungicidas } \\
\text { aéreos }\end{array}$} & Bravonil 720 SC & Clorotalonil & II & Cloronitrilos \\
\hline & Syllit $400 \mathrm{SC}$ & Dodine & II & Guanidinas \\
\hline & Instinct $750 \mathrm{EC}$ & Fenpropidin & II & Piperidinas \\
\hline & Siganex $60 \mathrm{SC}$ & Pyrimethanil & III & Anilinopirimidinas \\
\hline & Soprano $125 \mathrm{SC}$ & Epoxiconazol & II & Triazoles \\
\hline & Dithane $60 \mathrm{OF}$ & Mancozeb & III & Ditiocarbamatos \\
\hline & Silvacur Combi EC 300 & Tebucona/triadimenol & II & Triazoles \\
\hline & Volley $88 \mathrm{OL}$ & Fenpropimorf & IV & Morfolinas \\
\hline & Kurdo 250 EC & Epoxiconazole & III & Triazoles \\
\hline & Rubric 125 SC & Epoxiconazole & III & Triazoles \\
\hline & Odeon $720 \mathrm{SC}$ & Clorotalonil & III & Cloronitrilos \\
\hline & Impulse EC 800 & Spiroxamine & II & Spiroketalaminas \\
\hline & Centauro $720 \mathrm{SC}$ & Clorotalonil & II & Cloronitrilos \\
\hline & Duronil 720 SC & Clorotalonil & II & Cloronitrilos \\
\hline & Helcore $250 \mathrm{EC}$ & Difenoconazol & II & Triazoles \\
\hline & Bananin $86 \mathrm{OL}$ & Tridemorf & II & Morfolinas \\
\hline & Cumora $50 \mathrm{SC}$ & Boscalid & III & Piridina Carboxamidas \\
\hline & Calixin 75 EC & Tridemorf & II & Morfolinas \\
\hline & Banaclean 86 OL & Tridemorf & II & Morfolinas \\
\hline & Sico EC & Difenoconazol & III & Triazoles \\
\hline & Paladium 250 EC & Difenoconazol & III & Triazoles \\
\hline
\end{tabular}

como residuos de la corriente A4130, envases y contenedores de desechos que contienen sustancias incluidas en el Anexo I. En el sector bananero, estos residuos corresponden a los provenientes de laboratorios de análisis y control de calidad, así como del área de almacenamiento de materias primas o insumos químicos, los cuales se encuentran en las instalaciones de algunas fincas bananeras para efectuar control de la calidad del agua de proceso utilizada para el lavado y de los vertimientos líquidos de aguas residuales.

\section{DISCUSIÓN}

La presencia recurrente en fuentes de agua natural y/o suelo de diversos residuos peligrosos generados en las etapas de manejo de cultivo, cosecha y postcosecha de banano en la Zona Bananera, constituye un indicador de la deficiente gestión de éstos por parte de las empresas generadoras.

El impacto potencial negativo en el ambiente y en la salud humana resulta preocupante para el caso de residuos como bolsas de polietileno con aditivo 
CUADRO II. PROCESOS O ACTIVIDADES GENERADORAS DE RESIDUOS PELIGROSOS IDENTIFICADAS EN LAS FINCAS BANANERAS DEL MUNICIPIO ZONA BANANERA.

\begin{tabular}{ll}
$\begin{array}{l}\text { Procesos o } \\
\text { actividades }\end{array}$ & Descripción \\
\hline Y4 & Desechos resultantes de la producción, preparación y utilización de biocidas y productos fitofarmacéuticos. \\
\hline Y6 & Desechos resultantes de la producción, preparación y utilización de disolventes orgánicos. \\
\hline Y8 & Desechos de aceites minerales no aptos para el uso a que estaban destinados. \\
\hline Y9 & Mezclas y emulsiones de desechos de aceite y agua o hidrocarburos y agua. \\
\hline Y10 & $\begin{array}{l}\text { Sustancias y artículos de desecho que contengan o estén contaminados por bifenilos policlorados (PCB), terfenilos } \\
\text { policlorados (PCT) o bifenilos polibromados (PBB). }\end{array}$ \\
\hline Y12 & $\begin{array}{l}\text { Desechos resultantes de la producción, preparación y utilización de tintas, colorantes, pigmentos, pinturas, lacas o } \\
\text { barnices. }\end{array}$ \\
\hline Y18 & Residuos resultantes de las operaciones de eliminación de desechos industriales. \\
\hline Y31 & Desechos que contengan como constituyente plomo, compuestos de plomo. \\
\hline Y37 & Compuestos orgánicos de fósforo. \\
\hline Y45 & $\begin{array}{l}\text { Compuestos organohalogenados que no sean las sustancias mencionadas en categorías previas (e.g., Y39, Y41, Y42, } \\
\text { Y43, Y44). }\end{array}$ \\
\hline
\end{tabular}

CUADRO III. CORRIENTES DE RESIDUOS PELIGROSOS IDENTIFICADAS EN LAS FINCAS BANANERAS DEL MUNICIPIO ZONA BANANERA.

Corriente Descripción.

A1160 Acumuladores de plomos de desecho, enteros o triturados.

A1180 Montajes eléctricos y electrónicos de desecho o restos de éstos que contengan componentes como acumuladores y otras baterías incluidas en la lista $\mathrm{A}$, interruptores de mercurio, vidrios de tubos de rayos catódicos y otros vidrios activados y capacitadores de PCB, o contaminados con constituyentes del Anexo I (por ejemplo, cadmio, mercurio, plomo, bifenilo policlorado) en tal grado que posean alguna de las características del Anexo III (véase la entrada correspondiente en la lista B B1110).

A3020 Aceites minerales de desecho no aptos para el uso al que estaban destinados.

A3180 Desechos, sustancias y artículos que contienen, consisten o están contaminados con bifenilo policlorado (PCB), terfenilo policlorado (PCT), naftaleno policlorado (PCN) o bifenilo polibromado (PBB), o cualquier otro compuesto polibromado análogo, con una concentración igual o superior a $50 \mathrm{mg} / \mathrm{kg}^{1}$.

A4030 Desechos resultantes de la producción, la preparación y la utilización de biocidas y productos fitofarmacéuticos, con inclusión de desechos de plaguicidas y herbicidas que no respondan a las especificaciones, caducados, ${ }^{2}$ en desuso ${ }^{3}$ o no aptos para el uso previsto originalmente.

A4060 Desechos de mezclas y emulsiones de aceite y agua o de hidrocarburos y agua.

A4070 Desechos resultantes de la producción, preparación y utilización de tintas, colorantes, pigmentos, pinturas, lacas o barnices, con exclusión de los desechos especificados en la lista B.

A4130 Envases y contenedores de desechos que contienen sustancias incluidas en el Anexo I, en concentraciones suficientes como para mostrar las características peligrosas del Anexo III.

${ }^{1}$ Se considera que el nivel de $50 \mathrm{mg} / \mathrm{kg}$ es un nivel práctico internacional para todos los desechos; ${ }^{2}$ caducados significa no utilizados durante el periodo recomendado por el fabricante; ${ }^{3}$ se entenderá por plaguicida en desuso el plaguicida que: $a$ ) ha sido retirado del mercado por razones de salud o ambientales; $b$ ) ha sido prohibido o se ha cancelado su registro por decisión de la autoridad competente; c) ha perdido sus propiedades de control para los organismos previstos y no puede utilizarse para otros fines, ni puede ser fácilmente modificado para volver a ser útil; $d$ ) se ha contaminado con otros productos; $e$ ) se ha degradado debido a un almacenamiento inadecuado y prolongado, y no puede ser utilizado de acuerdo con las especificaciones e instrucciones indicadas en la etiqueta y por otra parte no puede ser reformulado; $f$ ) ha sufrido cambios químicos y/o físicos que puedan provocar efectos fitotóxicos en los cultivos o representa un peligro inaceptable para la salud de las personas o para el ambiente; $g$ ) ha sufrido pérdida inaceptable de su eficacia biológica por degradación de su ingrediente activo u otro cambio físico o químico; $h$ ) sus propiedades físicas han cambiado y por tanto no permite su aplicación en condiciones normales; $i$ ) sobrantes y remanentes que se pretenden descartar. 
clorpirifós/bifentrina y envases vacíos de agroquímicos, que con frecuencia se encuentran diseminados en puntos críticos del área de estudio, como los ríos Tucurinca, Sevilla y Riofrío.

En sí, los residuos de bolsas de polietileno generan una acumulación indefinida a causa de su nula o lenta biodegradación, aunado a que la bolsa deberá ser tratada como residuo peligroso si contiene aditivo clorpirifós/bifentrina (Torres-Rodríguez et al. 2013), con el agravante de que el clorpirifós (O,O-dietil O-3,5,6-tricloro-2-piridil fosforotioato) mantiene sus propiedades después de desechada la bolsa al momento de la cosecha (Castillo et al. 1997).

Así lo han documentado los estudios previos sobre la degradación hidrolítica del clorpirifós realizados por Macalady y Wolfe (1983), quienes reportaron que esta sustancia posee una vida media de 10 a 120 días en el agua y se transforma en 3,5,6-tricloro2-pyridinol (TCP) por mecanismos hidrolíticos. A su vez, Banks et al. (2005) afirmaron que el clorpirifós y sus productos de transformación pueden contaminar el recurso hídrico en zonas con gran actividad agrícola, lo que podría implicar un riesgo potencial para los seres humanos por exposición a este plaguicida y sus productos de degradación.

Los estudios de Narváez et al. (2012) indican que el pH básico del clorpirifós (superior a 9.0) favorece su rápida degradación y por lo tanto una vida media más corta. Sin embargo, resulta importante advertir que algunos productos de transformación pueden tener mayor capacidad de bioacumulación y ser más tóxicos que los compuestos primarios, como afirman Belfroid et al. (1998).

La inadecuada disposición de los envases vacíos de agroquímicos utilizados para el control fitosanitario en fincas bananeras, bien sea por fumigación aérea y/o aspersión manual, asociada con la categoría toxicológica del plaguicida, genera riesgos para la biodiversidad en los agroecosistemas, como afirman Mena-Espino y Couoh-Uicab (2015). En las condiciones actuales del área de estudio que es consecuente con lo indicado por Niti et al. (2013), su persistencia puede ocasionar acumulación en suelos, agua y organismos, con el agravante de la bioacumulación en plantas y posterior ingesta por animales (especialmente el ganado) o directamente por seres humanos.

\section{CONCLUSIONES}

Los residuos de plaguicidas asociados con la actividad de producción de banano de exportación en el municipio Zona Bananera, Departamento del Magdalena, se generaron principalmente en las etapas de manejo del cultivo, cosecha y postcosecha, con mayor diversidad de generación durante la cosecha, especialmente envases de herbicidas, fungicidas foliares y plaguicidas manuales, que en su mayoría se encuentran en la categoría toxicológica II (productos altamente tóxicos), además del registro de residuos de bolsas con aditivo clorpirifós y EPP contaminados.

La identificación de estos residuos peligrosos en una fase diagnóstica facilita la elaboración e implementación del Plan de Gestión de Residuos Peligrosos en Zona Bananera, asociado a las actividades productivas del banano de exportación, con miras a la gestión integral de los residuos peligrosos por parte de los generadores y al fortalecimiento de acciones que orienten el ejercicio de las funciones misionales por parte de los entes reguladores en materia ambiental.

\section{AGRADECIMIENTOS}

Los autores expresan su agradecimiento a la Corporación Autónoma Regional del Magdalena, a las cooperativas de recuperadores de residuos y a las fincas bananeras que apoyaron con el suministro de la información utilizada para la elaboración de este trabajo.

\section{REFERENCIAS}

Alcaldía Municipal Zona Bananera (2016). Plan de desarrollo municipal 2016-2019. Zona Bananera, Departamento del Magdalena, Colombia, 212 pp.

AUGURA (2017). Coyuntura bananera 2017. Asociación de Bananeros de Colombia. Informe. [en línea] http://www.augura.com.co/wp-content/uploads/2015/08/COYUNTURA-BANANERA-2017. pdf $10 / 07 / 2020$

Banks K.E., Hunter D.H. y Wachal D.J. (2005). Chlorpyrifos in surface waters before and after a federally mandated ban. Environ. Int. 31 (3), 351-356. https:// doi.org/10.1016/j.envint.2004.08.007

Belfroid A.C., van Drunen M., Beek M.A., Schrap S.M., van Gestel C.A. y van Hattum B. (1998). Relative risks of transformation products of pesticides for aquatic ecosystems. Sci. Total Environ. 222 (3), 167-183. https://doi.org/10.1016/S0048-9697(98)00298-8

Castillo L.E., de la Cruz E. y Ruepert C. (1997). Ecotoxicology and pesticides in tropical aquatic ecosystems of Central America. Environ. Toxicol. Chem. 16 (1), 41-51. https://doi.org/10.1002/etc.5620160104 
CORPAMAG (2016). Plan de acción institucional PAI 2016-2019: ¡Magdalena ambiental, compromiso de todos! Corporación Autónoma Regional del Magdalena. Santa Marta, Colombia, 133 pp.

CORPAMAG (2017). Plan de gestión integral de envases de agroquímicos, bolsas plásticas tratadas con aditivo clorpirifós y residuos postconsumo en el sector de Zona Bananera, Departamento del Magdalena. Contrato núm. 193 de 2016. Corporación Autónoma Regional del Magdalena. Santa Marta, Colombia, 53 pp.

DNP (2016). Ficha de caracterización territorial municipio de Zona Bananera. Departamento Nacional de Planeación [en línea]. https://terridata.dnp.gov.co/\#/ perfiles 07/08/2018

Gómez-Arroyo S., Martínez-Valenzuela C., CarbajalLópez Y., Martínez-Arroyo A., Calderón-Segura M.E., Villalobos-Pietrini R. y Waliszewski S. (2013). Riesgo genotóxico por la exposición ocupacional a plaguicidas en América Latina. Rev. Int. Contam. Ambie. 29, 159-180.

Guzmán-Cabrera A., Noa-Carrazana J.C., EscalanteRebolledo M.A. y Susan-Tepetlán P.V. (2017). Residualidad de plaguicidas en suelos dedicados al cultivo de banano dominico (Musa spp.) en Taplacoyan, Veracruz, y sus posibles efectos a la salud. Uvserva 4, 58-66.

Macalady D. y Wolfe N. (1983). New perspectives on the hydrolytic degradation of the organophorothioate insecticide chlorpyrifos. J. Agric. Food Chem. 31 (6), 1139-1147. https://doi.org/10.1021/jf00120a003

MADR (2016). Evaluaciones agropecuarias. Área, producción, rendimiento y participación municipal en el departamento por cultivo. Ficha técnica. Estadísticas. Ministerio de Agricultura y Desarrollo Rural, Colombia [en línea]. http://www.agronet.gov.co/estadistica/ Paginas/default.aspx 29/03/2017

MADS (2015). Decreto 1076 de 26 de mayo: Decreto único reglamentario del Sector Ambiente y Desarrollo Sostenible. Ministerio de Ambiente y Desarrollo Sostenible, Bogotá, Colombia, 654 pp.

Mena-Espino X. y Couoh-Uicab Y. (2015). Efectos de los plaguicidas utilizados para el control de la Sigatoka negra en plantaciones bananeras en México, así como su efecto en el ambiente y la salud pública. Tecnociencia Chihuahua 9 (2), 115-122.

Moreno J.M., Blanco C. y Mendoza R.J. (2009). Buenas prácticas agrícolas en el cultivo del banano en la Región del Magdalena: Reducción del escurrimiento de plaguicidas al Mar Caribe. GEF/PNUMA/REPCAR/ AUGURA, Medellín, Colombia, 56 pp.

Narváez J.F., Palacio J.A y Molina F.J. (2012). Persistencia de plaguicidas en el ambiente y su ecotoxicidad: una revisión de los procesos de degradación natural. Gestión y Ambiente 15 (3), 27-38.

Niti C., Sunita S., Kamlesh K. y Rakesh K. (2013). Bioremediation: An emerging technology for remediation of pesticides. Res. J. Chem. Environ. 17(4), 88-105.

Torres-Rodríguez A., Bernal-Vera M.E. y CastañoRamírez E. (2013). Evaluación ambiental de la práctica “embolsado" en plátano (Musa AAB Simmonds). Quindío, Colombia. Luna Azul 36, 91-109. 